\title{
Self-Sufficient Wind Turbine Condition Monitoring System
}

\author{
Rana N. Badran, Ihab Adly, and Hani Ghali
}

\begin{abstract}
Unpredictable failures of wind turbines and the associated costs; have contributed to the development of condition monitoring systems. The availability of ultra-low power devices has contributed in creating autonomous systems that operates on energy harvested from the surrounding environment. This research aims to develop a design of a self-sufficient condition monitoring system, that is able to run on renewable energy. Energy is harvested from an electromagnetic energy harvester that harnesses the rotational motion of the wind turbine blades and converts it to useful power. After conditioning and storing the harvested energy, the maximum available power is $435 \mathrm{~mW}$. The energy harvested is used to charge a lithium ion battery for backup as well as power the condition monitoring system. The system is validated by both simulation models and experimental measurements on a wind turbine prototype model.
\end{abstract}

Index Terms - Condition monitoring, energy harvesting, signal conditioning, ultra-low power.

\section{INTRODUCTION}

Wind turbines are composed of different mechanical and electrical structures that are continuously exposed to varying operating conditions [1]. Without properly monitoring the performance of the wind turbine, costs of operation and maintenance can greatly increase [2], [3]. The most common condition monitoring technique is vibration analysis [4]. It is typically used to monitor the fundamental components of a wind turbine, such as: the gearbox, and generator [5]. Professionals can then analyse the vibration data and identify the exact fault/damage that is detected [6], [7].

Currently there is a tendency to acquire ambient energy for powering sensors and electronic devices used in remote locations [8], [9]. However, a major restriction in the field of energy harvesting is the fact that the energy generated by harvesting devices is extremely small to directly power most electronics [10]. To address this problem, efficient, innovative and adaptive methods need to be implemented. Ultra-low power and energy efficient sensors and microcontrollers are the key technologies that will allow energy harvesting to become a source of power for electronic

Manuscript received May 4, 2017; revised August 2, 2017.

R. N. Badran is with the Electrical Engineering Department, The British University in Egypt, Cairo 11837, Egypt (e-mail: rana.badran@bue.edu.eg).

I. Adly is with Tegrom, Cairo 11361, Egypt (e-mail: ihab.adly@tegrom.com).

H. Ghali is with the Electrical Engineering Department, University of Ain Shams, Cairo 11517, Egypt (e-mail: hani.amin@bue.edu.eg). systems.

This research aims to design a self-sufficient condition monitoring system for wind turbines that is powered solely using renewable energy solutions. The energy is harvested by means of an electromagnetic energy harvester installed inside the blades. The harvested power is conditioned to provide a stable output for powering up the electronic components of the condition monitoring system. The system uses an accelerometer, microcontroller and wireless module to measure the vibrations of the gearbox and/or generator and send the data wirelessly for inspection. System simulation and design are explained in details in this paper. The system is validated by both simulation and experimental data on a wind turbine prototype model.

\section{SELF-Sufficient Condition Monitoring System DESIGN}

As shown in Fig. 1, the electromagnetic energy harvester will be installed inside the blades of the wind turbine, this setup was first introduced by Joyce [11]. The harvester is part of the power module that will be used to power up the electronic components of the condition monitoring system.

\section{A. Power Module}

The power module in Fig. 1 is responsible for converting the pulses generated by the electromagnetic energy harvester to useful DC supply. This can be done by rectification, boosting, regulation and storage of the power. The power module includes three components. They are responsible for the following: electromagnetic energy harvesting, signal conditioning and energy storage.

The electromagnetic energy harvester is composed of a closed tube containing a magnet, and on the outside of the tube is a wound up coil of multiple turns. The electromagnetic harvester will be installed inside the blade to prevent any aerodynamic imbalance. Each of the three blades will have the same setup inside. A simulation model for the electromagnetic harvester was built on COMSOL Multiphysics, simulation results are shown in Fig. 2 and the computed open circuit voltage is shown in Fig. 3. Moreover, in order to test the actual output voltage of the harvesting system, a wind turbine prototype model was built for experimental testing.

In the prototype model, the motion of the blades as a result of the wind is simulated by using an electric DC motor. Therefore, the blades along with the hub were installed to a motor shaft. The complete setup is shown in Fig. 4 and the prototype specifications are listed in Table I. After installing the blades, with the electromagnetic harvesters, the blades 
were made to rotate at $16 \mathrm{RPM}$, which is an average range for a $1 \mathrm{MW}$ wind turbine. The electromagnetic harvester was fixed on top of the blades since the blades of the model are not hollow, however; in an actual wind turbine, the harvester should be fixed inside the blades. The coils of all the three tubes were connected in series. The open circuit voltage of the electromagnetic harvester is shown in Fig. 5. The total impedance of the coils is around $36 \Omega$, therefore the harvester would be able to supply a maximum current of $89 \mathrm{~mA}$.

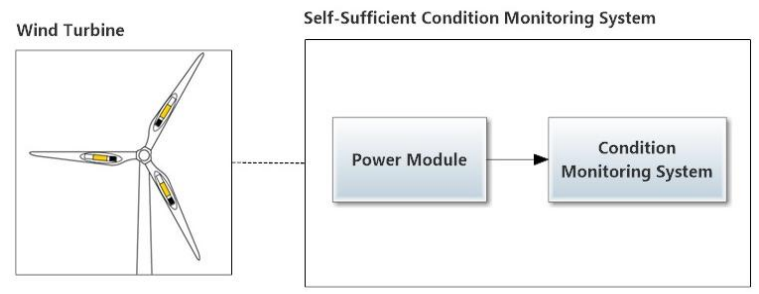

Fig. 1. Architecture of the self-sufficient condition monitoring system.

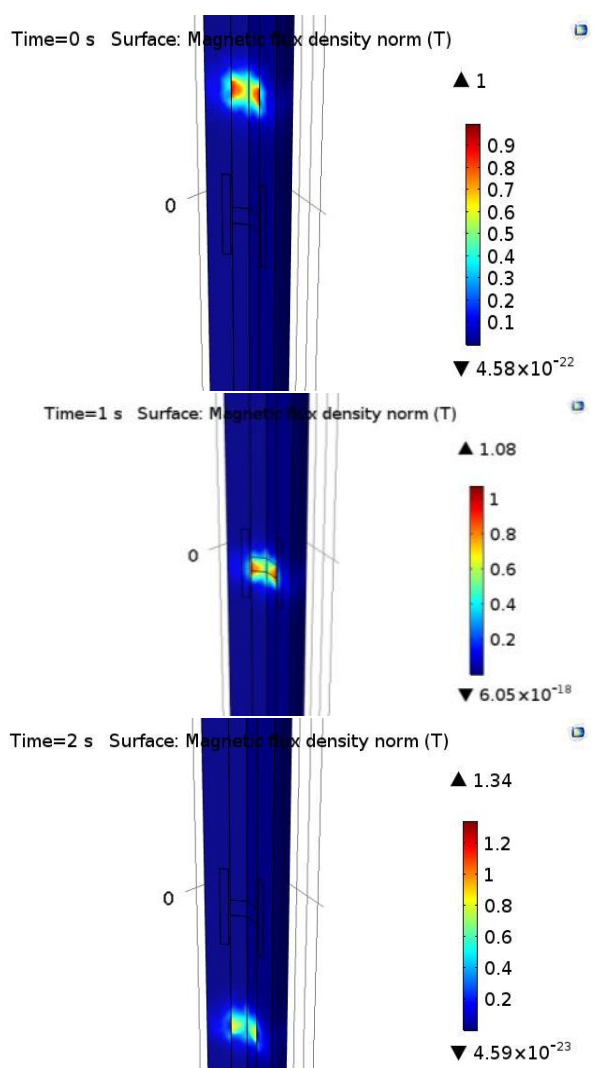

Fig. 2. Magnetic flux density that is computed from COMSOL Multiphysics as the magnet moves inside the tube.

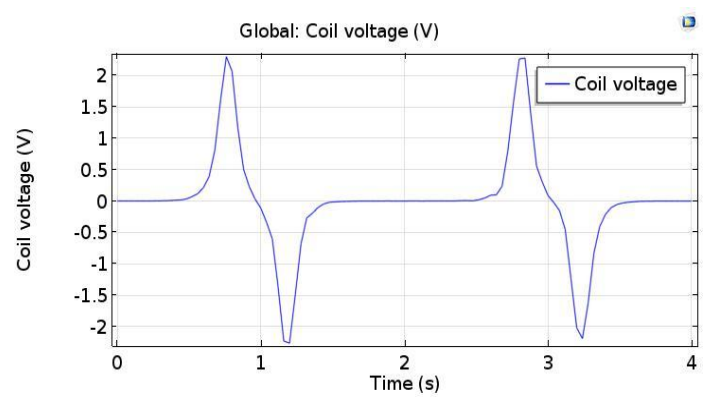

Fig. 3. Results for open circuit voltage that is computed from COMSOL Multiphysics.

For signal conditioning, there are three necessary stages to rectify, boost and regulate the raw harvested power from the electromagnetic energy harvester. First step, is designing a rectifying circuit. To minimize the voltage-drop on the diodes, schottky diodes 1N5817 were used since they have a low forward voltage of around $0.3 \mathrm{~V}$. The capacitor chosen is $1000 \mu \mathrm{F}$. The open circuit voltage of the rectifier is $3.5 \mathrm{~V}$, shown in Fig. 6(a), with a maximum current output of $40 \mathrm{~mA}$.

The second stage is a micro power step-up low power voltage booster module that needs to be used to increase the voltage level of the acquired signal. For this stage the EH4205 from Advanced Linear Devices was used. The reason behind choosing this specific model was its excellent power requirements. The EH4205 has the capability of self-powering using input power as low as $200 \mu \mathrm{W}$. The output voltage of the booster is shown in Fig. 6(b).

TABLE I: PROTOTYPE SPECIFICATIONS

\begin{tabular}{ll}
\hline \hline Part & Description \\
\hline Copper wire turns per tube & $700 \mathrm{turns}$ \\
Thickness of copper wire & $0.3 \mathrm{~mm}$ diameter \\
Length of the blade & $50 \mathrm{~cm}$ \\
Magnet dimensions & $2 \mathrm{~cm}$ diameter $\times 2 \mathrm{~cm}$ height \\
Magnet surface field & 6619 Gauss \\
Tube dimensions & $2.5 \mathrm{~cm}$ diameter $\times 20 \mathrm{~cm}$ height \\
Magnet weight & $18 \mathrm{~g}$ \\
\hline \hline
\end{tabular}

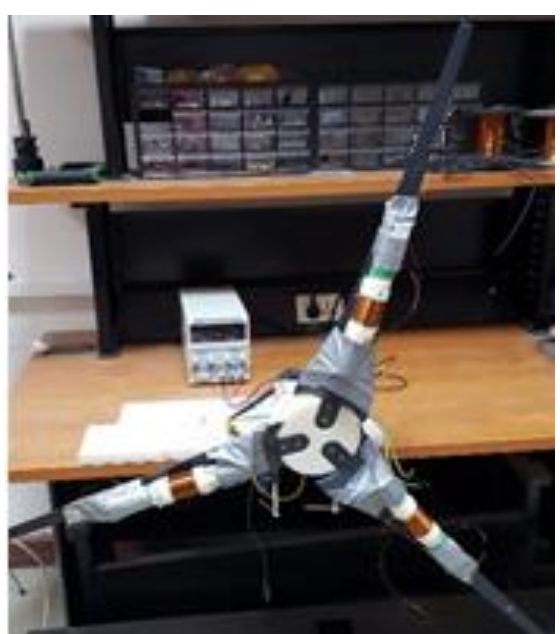

Fig. 4. Wind turbine prototype model with the electromagnetic energy harvester installed.

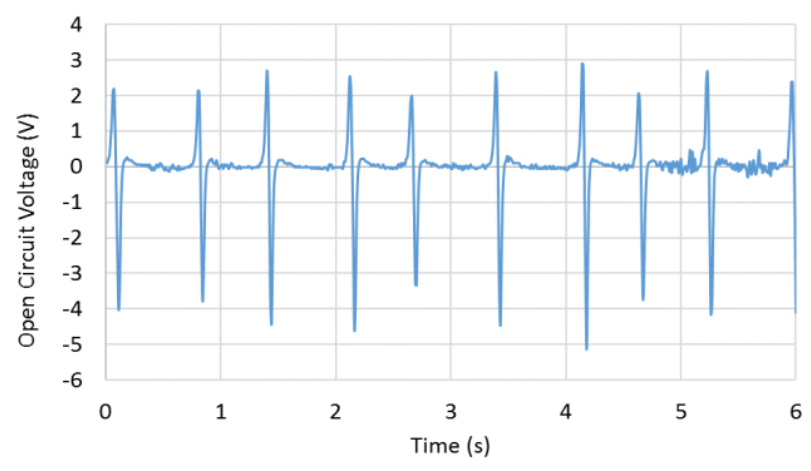

Fig. 5. Output voltage of energy harvester installed on the prototype model.

The third and final stage aims to convert the voltage pulses to a steady DC output voltage of minimum $3.3 \mathrm{~V}$. To achieve this, the EH301 energy harvester from advanced linear devices was used. When an energy source starts to inject voltage pulses of $6 \mathrm{~V}$ or higher into the inputs of an EH301, the energy is collected, and stored onto an internal storage 
capacitor bank. The output voltage of this stage is a steady 5.24 V DC, shown in Fig. 6(c). The output power of this stage is calculated and is equal to $435 \mathrm{~mW}$.

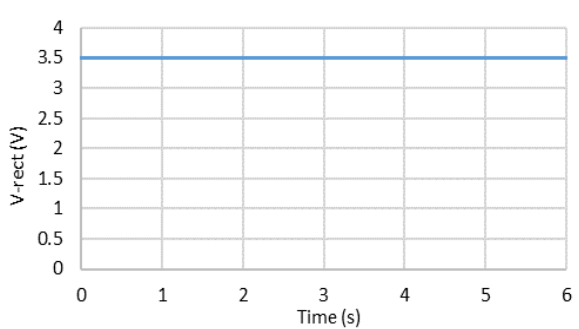

(a)

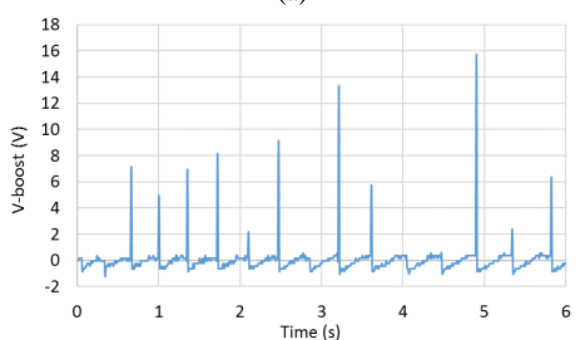

(b)

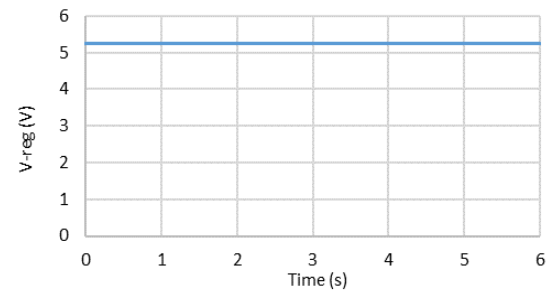

(c)

Fig. 6. Output voltage measured at (a) stage one (b) stage two (c) stage three

After signal conditioning, the next step is storing the harvested power by charging a lithium ion battery. This is a necessary step in order to guarantee continuous power supply in case of down times and/or low harvested power. To implement this, a very low-power battery charger using the MCP73831T chip is chosen to charge a polymer lithium ion Single Cell Battery 3.7 V, $300 \mathrm{mAh}$. The system was able to successfully charge the battery from $30 \%$ to $85 \%$ in 1.5 hours.

\section{B. Condition Monitoring System}

Fig. 7 shows the design and hardware setup of the condition monitoring system. The system will be installed on the gearbox or the generator of the wind turbine. The system components and power requirements are shown in Table II.

All the modules in Table II were chosen for their high performance and their ultra-low power characteristics. In order to guarantee that the designed power module will supply enough power to the condition monitoring system, the power consumption of each component was analyzed and noted in Table II. Since the total power supplied by the power module is $435 \mathrm{~mW}$, therefore it is sufficient for powering the condition monitoring system.

Fig. 8 shows the circuit connections of the condition monitoring system components as simulated on Proteus. The microcontroller is directly connected to the power module via the raw input terminal. To set up the interface between the sensor and microcontroller, I2C protocol was used. This is a multi-master protocol that uses two signal lines, which are the "serial data" (SDA) and "Serial Clock" (SCL). The microcontroller was programed to interface the sensor and read the data in bytes ( 2 bytes for each axis). In order to start reading vibration data from the accelerometer the following was implemented in the code:

- Set up the accelerometer's initialization registers (Data Format register, Power Control register, First axis-acceleration data register)

- Set up the accelerometer for continuous data output

- Set up the accelerometer to full resolution

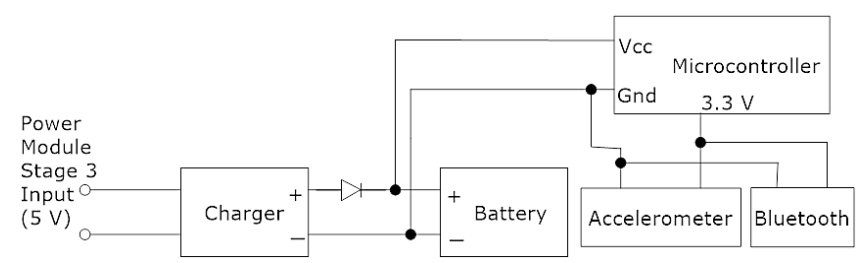

Fig. 7. Power connections for the condition monitoring system.

TABLE II: POWER CONSUMPTION OF CONDITION MONITORING SYSTEM COMPONENTS

\begin{tabular}{ll}
\hline \hline Component & Maximum power consumption \\
\hline ATMega328 microcontroller & $66 \mathrm{~mW}$ \\
HC-05 Bluetooth module & $147 \mu \mathrm{W}$ \\
ADXL345 accelerometer & $23 \mu \mathrm{W}$ \\
\hline \hline
\end{tabular}

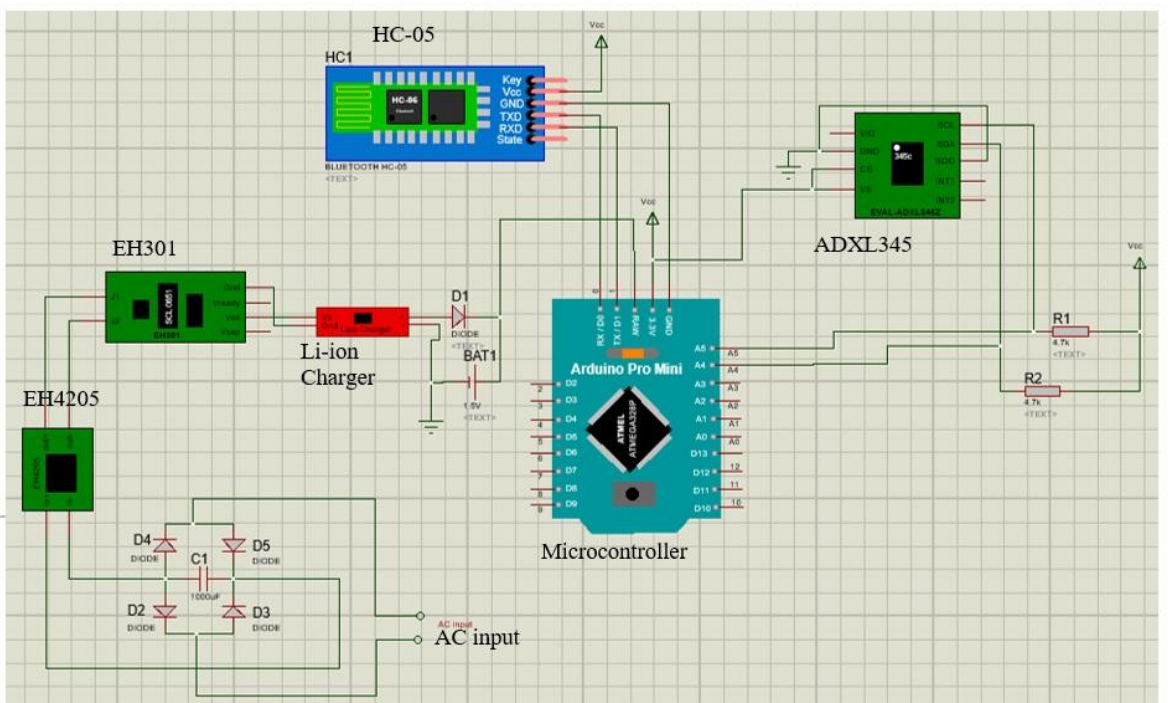

Fig. 8. Complete schemtaic diagram for the self suffcient condition monitoring system. 


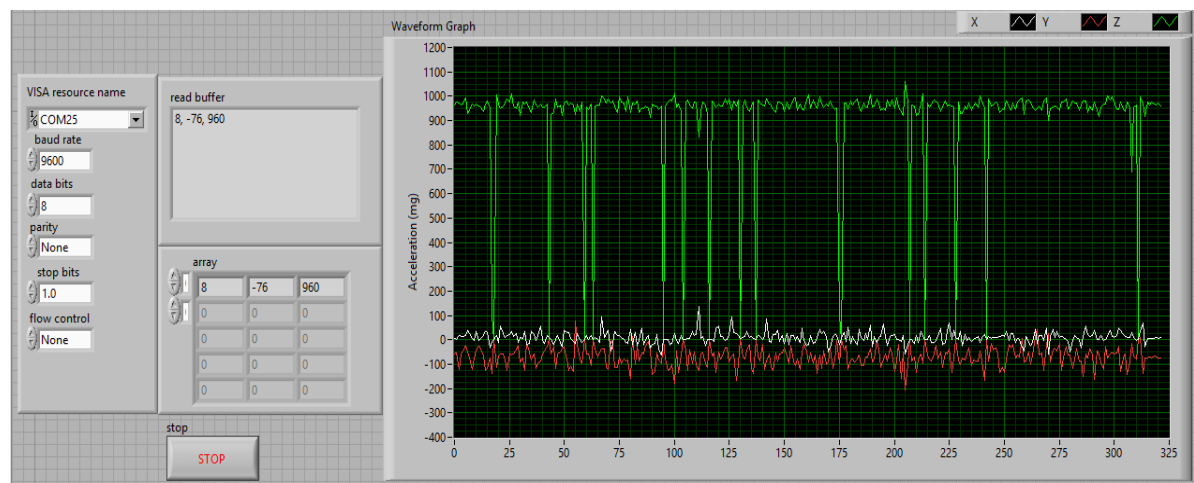

Fig. 9. Labview graphical user interface of the program that recieves the vibration data from the monitoring system

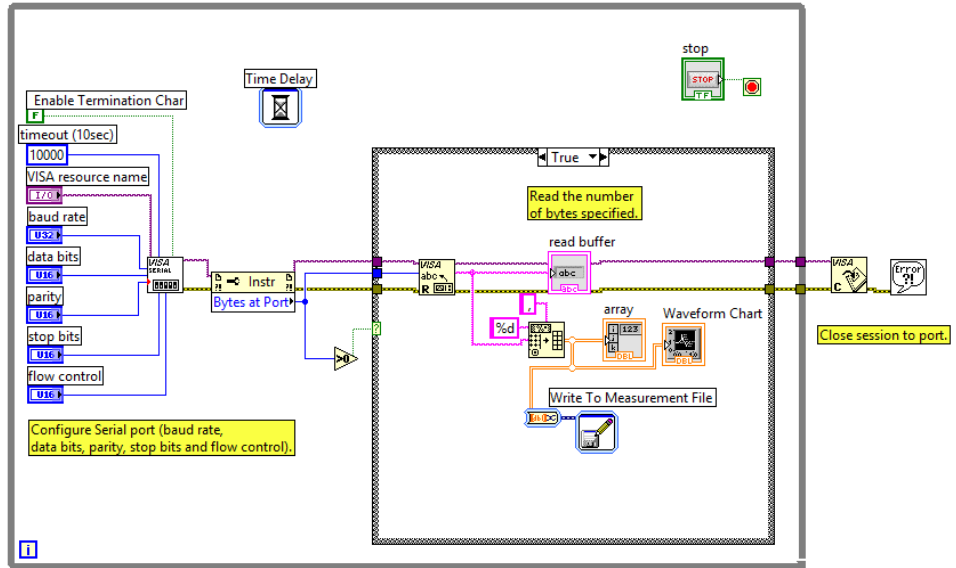

Fig. 10. Labview block code of the program that recieves the vibration data from the monitoring system

The microcontroller was connected to the Bluetooth Module via the Tx and Rx ports where they can communicate using serial interface. The accelerometer has 13-bit resolution and a wide vibration measurement range $( \pm 16 \mathrm{~g})$. For lower power operation the accelerometer was set on standby mode when there is no activity, this limits current consumption to $0.1 \mu \mathrm{A}$. The sampling rate of the accelerometer is $200 \mathrm{~Hz}$. The readings of the accelerometer are sent via Bluetooth to a nearby computer device for analyzing the condition of the wind turbine. The communications time is $9600 \mathrm{bits} / \mathrm{s}$, data bit: 8, stop bit: 1, no parity bit, no Flow Control. After supplying power to the Bluetooth module, the device enters communication mode. Next step is to pair it to the computer that is to receive the vibration data. As soon as the device is paired, the Bluetooth will enter order-response work mode. After the Bluetooth is paired to the computer and as soon as the serial port is opened the vibration data starts logging.

\section{EVALUATION}

After successfully designing the power module, and the condition monitoring system, it is time to validate that the self-sufficient condition monitoring system is capable of measuring vibration data and sending them wirelessly to a nearby computer. To achieve this, a program was designed on LabView software to receive the data bytes sent by the accelerometer and display it on a real time graphical chart. The interface of the program is shown in Fig. 9 and the block code of the program is shown in Fig. 10.

\section{CONCLUSIONS}

Results are very promising as the power module was able to power all the required electrical components for the monitoring process. This includes the vibration sensor, microcontroller and the wireless module. Also the accelerometer was able to send the vibration readings real-time to a nearby computer. Results were displayed on a program created on LabView, the program can analyze, save and process that received data.

Future work requires studying the implementation of this system on an actual wind turbine, while studying the effect of the weight of the electromagnetic energy harvester on the wind turbine blades.

\section{REFERENCES}

[1] R. W. Hyers, J. G. McGowan, K. L. Sullivan, J. F. Manwell, and B. C Syrett, "Condition monitoring and prognosis of utility scale wind turbines," Journal of Energy Materials, vol. 1, no. 3, pp. 187-203, 2006.

[2] I. Antoniadou, G. Manson, W. J. Staszewski, T. Barszcz, and K Worden, "A time-frequency analysis approach for condition monitoring of a wind turbine gearbox under varying load conditions," Journal of Mechanical Systems and Signal Processing, vols. 64-65, pp. 188-216, 2015.

[3] J. Ribrant, "Reliability performance and maintenance - A survey of failures in wind power systems," MS. thesis, Dept. Elect. Eng., KTH Univ., Stockholm, Sweden, 2006.

[4] M. Nie and L. Wang, "Review of condition monitoring and fault diagnosis technologies for wind turbine gearbox," Journal of Procedia CIRP, vol. 11, pp. 287-290, 2013.

[5] S. Veers and P. Sheng, "Wind turbine drivetrain condition monitoring - An overview," in Proc. Mechanical Failures Prevention Group: Applied Systems Health Management Conference, Virginia, 2011.

[6] P. Tchakoua, R. Wamkeue, M. Ouhrouche, F. Slaoui-Hasnaoui, T. A. Tameghe, and G. Ekemb, "Wind turbine condition monitoring: State-of-the-art review, new trends, and future challenges," Journal of Energies, vol. 7, pp. 2595-2630, 2014.

[7] J. Paulo and P. D. Gaspar, "Review and future trend of energy harvesting methods for portable medical devices," in Proc. World Congress on Engineering, London, U.K, 2010. 
[8] A. Albarbar, A. Badri, J. K. Sinha, and A. Starr, "Performance evaluation of MEMS accelerometers," Journal of Measurement, vol. 42, no. 5, pp. 790-795, 2009.

[9] J. Igba, K. Alemzadeh, C. Durugbo, and E. T. Eiriksson, "Analysing RMS and peak values of vibration signals for condition monitoring of wind turbine gearboxes," Journal of Renewable Energy, vol. 91, pp. 90-106, 2016.

[10] R. Saidur, N. Rahim, M. Islam, and K. Solangi, "Environmental impact of wind energy," Journal of Renewable and Sustainable Energy Reviews, vol. 15, pp. 2423-2430, 2011.

[11] B. S. Joyce, "Development of an electromagnetic energy harvester for monitoring wind turbine blades," MS. thesis, Dept. Mech. Eng., Virginia Polytechnic Institute and State Univ., Virginia, 2011.

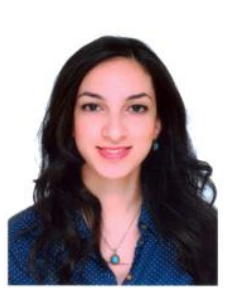

Rana N. Badran was born in Cairo, Egypt in 1990 In June 2012, she received her B.Sc. degree in electronics and communication engineering from the British University in Egypt (BUE), Cairo, Egypt. Her graduation project was about piezoelectric energy harvesting systems. Currently, she is pursuing her M.Sc. degree in renewable energy from the British University in Egypt.

Since September 2012, she has been working as a teaching assistant in the Electronics and Communications Department, Faculty of Engineering, the British University in Egypt. Her research interests are in the fields of renewable energy, energy harvesting and computer engineering.

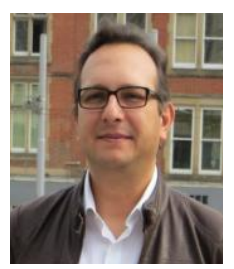

Ihab Adly received the B.Sc., M.Sc., Ph.D. degrees in electrical engineering from Ain Shams University (ASU), Cairo, Egypt in 1996, 2005 and 2010 respectively.

From 2006 to date, he is actively working in the industry through many research projects in different sectors mainly; oil \& gas, renewable energy and military communication sector. He then joined the Electronics and Communication Engineering Department, Faculty of Engineering, British University in Egypt as a lecturer from 2012 till end of June 2014. He is currently the PI of a research project funded through the EU to develop a Smart Wireless LED driver for Public Lighting expected to end in 2015 From 2010 to 2014, he has been working as a lecturer in the British University in Egypt, teaching Digital Design, Computer Architecture, VLSI Technology, and Embedded Systems courses.

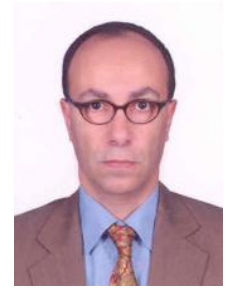

Hani Ghali earned his B.Sc. in electronics and computer engineering in 1983 from the Faculty of Engineering, Ain-Shams University (ASU), Cairo, Egypt. He got the M.Sc. in 1988 from the same University in "Analysis and Design of Fine-Line Circulator". In 1989, he joined the National Institute of Applied Sciences, Rennes, France studying towards his $\mathrm{PhD}$ degree in planar electromagnetic systems, where in 1992 he earned his Ph.D. in "Dynamic Analysis of Two and Three Dimensional Discontinuities and Interconnections using the Integral Equation Technique”.

During the period 1983-1988 he was appointed as a teaching assistant in the Electronics and Communications Engineering Department, Faculty of Engineering, Ain-Shams University, Cairo, Egypt. Since 1992, he was with the Electronics and Communications Engineering Department, Faculty of Engineering, Ain-Shams University, Cairo, Egypt as an assistant professor, then as an associate professor and finally, in March 2005, as a full professor. His field of research includes topics related to energy with emphasis on PV; use of Carbon Nanotube for Solar-Energy Harvesting, Photovoltaics (PV) Piezoelectric Energy Harvesting System and Nanowire-Based Photovoltaic (PV) Devices. 